\title{
Experimental study of bubble sweep-down in wave and current circulating tank: Part I-Experimental set-up and observed phenomena
}

\author{
Delacroix Sylvain ${ }^{1}$, Germain Grégory ${ }^{1,{ }^{*}}$, Gaurier Benoit ${ }^{1}$, Billard Jean-Yves ${ }^{2}$ \\ 1 IFREMER, Marine Structures Laboratory, 150 Quai Gambetta, 62321 Boulogne-sur-Mer, France \\ 2 IRENAV, Ecole Navale, 29240 Brest, France \\ *Corresponding author : Grégory Germain, email address : gregory.germain@ifremer.fr \\ iyvesbilla@numericable.fr
}

\begin{abstract}
:
Bubble sweep-down is a significant issue for the oceanographic vessels, which affect the acoustic surveys. Experimental trials, carried out in the Ifremer wave and current circulating tank on a 1/30 model of the Pourquoi pas?, are presented. The results demonstrate that this kind of experimental facility is well suited to study the phenomenon of bubble sweep-down encountered around the bow of a ship under specific conditions. From these results, two kinds of bubble clouds formation have been observed and analysed: bubble clouds generated by vortex shedding and breaking waves. The vortex shedding bubble clouds appear randomly in all the configurations tested, even without waves or motions. This phenomenon is due to the interaction between the turbulent flow and the specific bow shape of the Pourquoi pas?. On the other hand, the breaking wave clouds appear in the presence of relative motions between the free surface and the bow ship and more significantly under wave sollicitations. A complementary paper presents a parametric study carried out to quantify the influence of the test conditions.
\end{abstract}

\section{Highlights}

An experimental set-up has been developed to reproduce the ship behaviour under waves sollicitation. The bubble sweep-down phenomenon is experimentally studied. The waves and motions effects on bubble sweep-down phenomenon is presented. Vortex shedding and breaking waves clouds are characterized. The correlation between the free surface deformation and the air entrainment is highlighted.

Keywords : Bubble sweep-down, Experimental trials, Ship motions, Bow waves 


\section{Introduction}

The ocean is an opaque environment where the light only propagates over several meters. The observation of fisheries or seabed topography are consequently complex and require the use of acoustic waves able to propagate over several kilometres and carry back information after reflection on an obstacle. Specific acoustic equipments, single or multi-beam echosounders, are used on oceanographic vessels for many varieties of research. These echosounders are calibrated to work precisely in seawater, independently of the water temperature and density. Ship motions can also be compensated. However the acoustic waves can be strongly disrupted (reflected or 
absorbed) if air bubbles are present in the sea layer under the hull. This phenomenon has affected the acoustic surveys for many years (Dalen and Løvik (1981); New (1992)) and is still a significant issue (Shabangu et al. (2014)).

The bubble distribution in the upper ocean is caused by two main sources. Natural aeration is mostly due to the entrapment of air by breaking waves and rain impacting on the ocean surface. Several works have been dedicated to this topic, motivated by gas exchanges between the ocean and the atmosphere. Chanson and Cummings (1994) described a theoretical method to predict the size and the depth of entrained bubbles. Deane and Stokes $(1999,2002)$ developed an optical system able to record bubbles of radii $>200 \mu \mathrm{m}$. Trevorrow (2003) used a high frequency sonar for the measurements of near-surface bubble plumes in the upper ocean layer. All these studies demonstrated that micro-bubbles are dispersed by turbulence to depths of several meters (up to $25 \mathrm{~m}$ according to Trevorrow) in bad weather conditions. On the other hand, the ship bow wave generates a significant aeration, and the flow around the hull may carry self-generated bubbles under sonar locations. This phenomenon of bubble sweep-down, favoured by ship motions, must be prevented as much as possible in order to ensure a good productivity for a ship dedicated to acoustic survey.

Rolland and Clark (2010) have compared several solutions adopted to avoid bubble sweepdown. Gondolas, fairings and bubble fences can effectively reduce bubble sweep-down but generally significantly increase the ship resistance. During the design of the future Research Vessels Discovery and Sonne, particular attention was given to the bubble sweep-down issue (Cooper (2012); Von Bröckel (2014)). From past experiences, general conclusions are given as the benefit of a deep draft, or to avoid bulbous bow. However, considering all these elements does not guarantee the reduction of bubble sweep-down. Indeed the Research Vessel Pourquoi pas? of Ifremer fleet, although equipped with a gondola, has encountered significant bubble sweep-down interferences since the first sea trials in 2005 (Delacroix (2016)).

The tools allowing the study of this phenomenon are limited. Numerical simulations of this kind of diphasic problem are still difficult to achieve because of the scale difference between bubbles production, governed by surface tension, and the flow all around the hull. Moraga et al. (2008) developed a physically-based model that locates the region of high void fraction. The model uses the bubble size distribution measured by Deane and Stokes (2002) to simulate the bubbly flow around the hull of the research vessel Athena with the prediction of air bubble locations. Ma et al. (2011) describe a subgrid model coupled with a two-fluid Reynolds Average Navier Stokes (RANS) bubbly flow model, and obtain the first quantitative numerical prediction of void fraction distributions around a full-scale surface ship. Lately Carrica et al. (2012) and Castro et al. (2014) improved the air entrainment model, taking into account turbulent and cavity free surface bubble entrainment. All these investigations have been performed in calm seas, without ship movements. To this point therefore, numerical studies used for bubble sweepdown consideration during ship designs are only based on the analysis of streamlines without waves and motions. The effect of different sea state is investigated by model test in towing tank. However, the generation of bubbles is usually low or non-existent around the model because of scale effects, and the evolution of the bubbles is simulated by dye injection at the bow vicinity. Conclusions of the these kind of tests are not always verified at sea (Pourquoi pas? experience 
and Von Bröckel (2014)). Specific experiments have already been developped to study the bow wave geometry (Noblesse et al., 2008, 2013; Delhommeau et al., 2009) and the resulting air entrainment (Waniewski et al., 2001; Shakeri et al., 2009; Tavakolinejad, 2010), but these studies focused on steady bow wave in calm seas for thin and fast ships. The influence of sea states and motions have not been considered.

We propose here an original experimental method under which the phenomenon can be studied. This method is based on the simulation, in a wave and current circulating tank, of sea conditions encountered by the French oceanographic vessel Pourquoi pas? during which bubble sweep-down was observed. We present in the first part of this paper the details of the experimental set-up and the two kinds of aeration phenomenon encountered by this way, while the analysis of the bubble clouds is described in the second part of the article.

\section{Experimental set-up}

This section aims at giving a thorough description of the experimental set-up and measurement facilities used for this study. The Pourquoi pas? is operated by Ifremer and the French Ministry of Defence, primarily for hydrography, submarine cartography and seabed characterization. Its main characteristics are a length between perpendiculars $L_{p p}=94 \mathrm{~m}$, a beam $B=20 \mathrm{~m}$ and a draft $T=5.45 \mathrm{~m}$ in operation. This vessel has been chosen for the considerable database of sea campaigns which is easily accessible, and the significant effect of bubble sweep-down on the acoustic equipments.

\subsection{Wave and current circulating tank facilities}

This experimental technique has been developed in the wave and current circulating tank of Ifremer (in Boulogne-sur-mer, France). In such facility, the model is fixed and the ship velocity is simulated by the current. The tank working section is $18 \mathrm{~m}$ long by $4 \mathrm{~m}$ wide and $2 \mathrm{~m}$ deep (see figure 1). Regarding scale effects, the model must be large enough to be able to observe bubble sweep-down. Therefore, the maximum scale have been chosen, taking into account the tank characteristics. A 1/30 model of the Pourquoi pas? allows to generate trials conditions with relatively low blockage and reflection effects in the bow vicinity. That scale corresponds to a ship model of $3.13 \mathrm{~m}$ length between perpendiculars (Lpp), $0.67 \mathrm{~m}$ beam and $0.18 \mathrm{~m}$ draft (see figure 2).

The circulating tank is equipped with a wave generator composed of 8 independent displacement paddles each $0.5 \mathrm{~m}$ wide and $0.5 \mathrm{~m}$ deep, which can be easily moved between an upstream or a downstream surface position in order to create waves propagating with or against the current. This wave generator enables the production of regular and irregular waves with a frequency range between $f=0.5-2 \mathrm{~Hz}$ and a maximum amplitude of $A=280 \mathrm{~mm}$ with a current up to $0.8 \mathrm{~m} / \mathrm{s}$. Measurements revealed that the resulting reflection coefficient is lower than $12 \%$ for all the usual wave frequencies and amplitudes. More details about the flume tank can be found in Gaurier et al. (2013) and Bouhoubeiny et al. (2014). The free surface elevation is measured by the mean of a wave gauge system with the wave probe situated $1 \mathrm{~m}$ upstream from the model bow. 


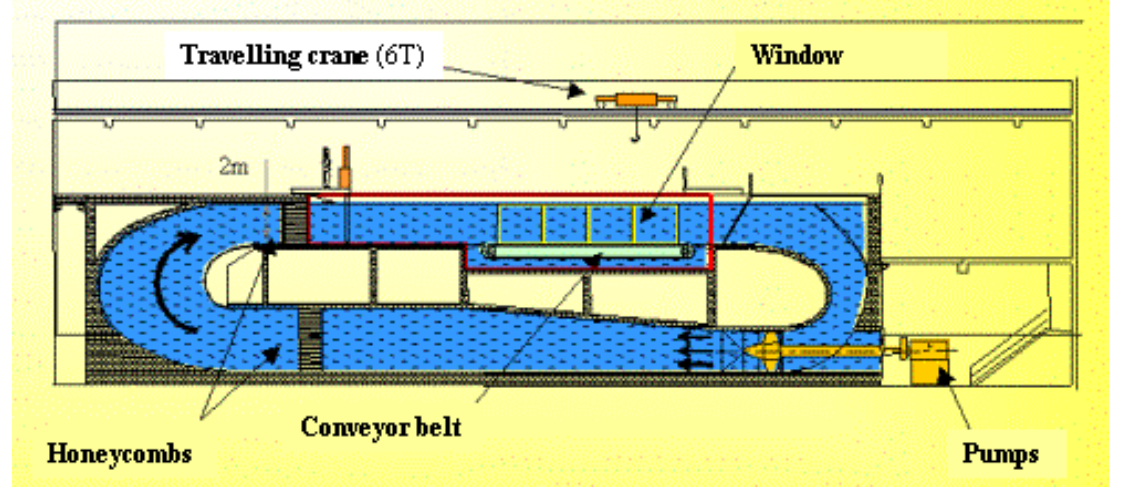

Figure 1: Schematic view of the wave and current circulating tank

When the wave generator is located upstream, there is a high turbulent intensity (TI) in the incoming flow. $T I=15 \%$ in this configuration, close to sea turbulence, while without the wave generator, the turbulent intensity is $T I=3 \%$, with:

$$
T I=\sqrt{\frac{1}{3} \frac{u^{\prime 2}+v^{\prime 2}+w^{\prime 2}}{\overline{u^{2}}+\overline{v^{2}}+\overline{w^{2}}}}
$$

Thanks to the Reynolds decomposition, $U=\bar{u}+u^{\prime}$ where $\bar{u}$ is the mean value and $u^{\prime}$ the fluctuating part of the current velocity $U$. The corresponding data have been measured with a 2D Laser Doppler Velocimetry system in two steps to acquire the three velocity components.

The major difference between this configuration and classic towing tank tests, during which models are free to heave and pitch, is that the ship motions are here forced by a motion generator system (hexapod).

This system allows to generate any motions in the 6 degrees of freedom. It is not necessary then to have a balanced ship model in terms of displacement, inertia and center of gravity, which greatly facilitates the design and construction of the scale model. The use of the motion generator also enables to study independently the effect of waves and motions on bubbles production. Above all, in this configuration it is possible to work with the front part only ( $1 / 3$ of the model). This possibility is very interesting in a relatively small circulating tank where perturbations comming from the model stern motions are indeed avoided.

The two different configurations are shown on figure 2. Measurements of the ship resistance were performed on the full model configuration. It has been shown that the flow in the circulating tank is representative of the real scale flow as in the classic towing tanks (Delacroix, 2015). After verification that the tests with the front part only have no influence on the flow in the bow region, all the study of bubble sweep-down have been undertaken in this configuration, in order to suppress the waves generated by the relatively flat end part of the Pourquoi pas? model.

The ship model motions are measured by the mean of the hexapod real time measurement system, with a precision of less than $0.1 \mathrm{~mm}$ and $0.01^{\circ}$.

The computer program FREDYN has been used for the calculation of the ship motions taking into account the experimental conditions. In this program, a nonlinear strip theory approach is used to compute the hydrodynamic forces acting on the hull (De Kat and Paulling (2001)). 

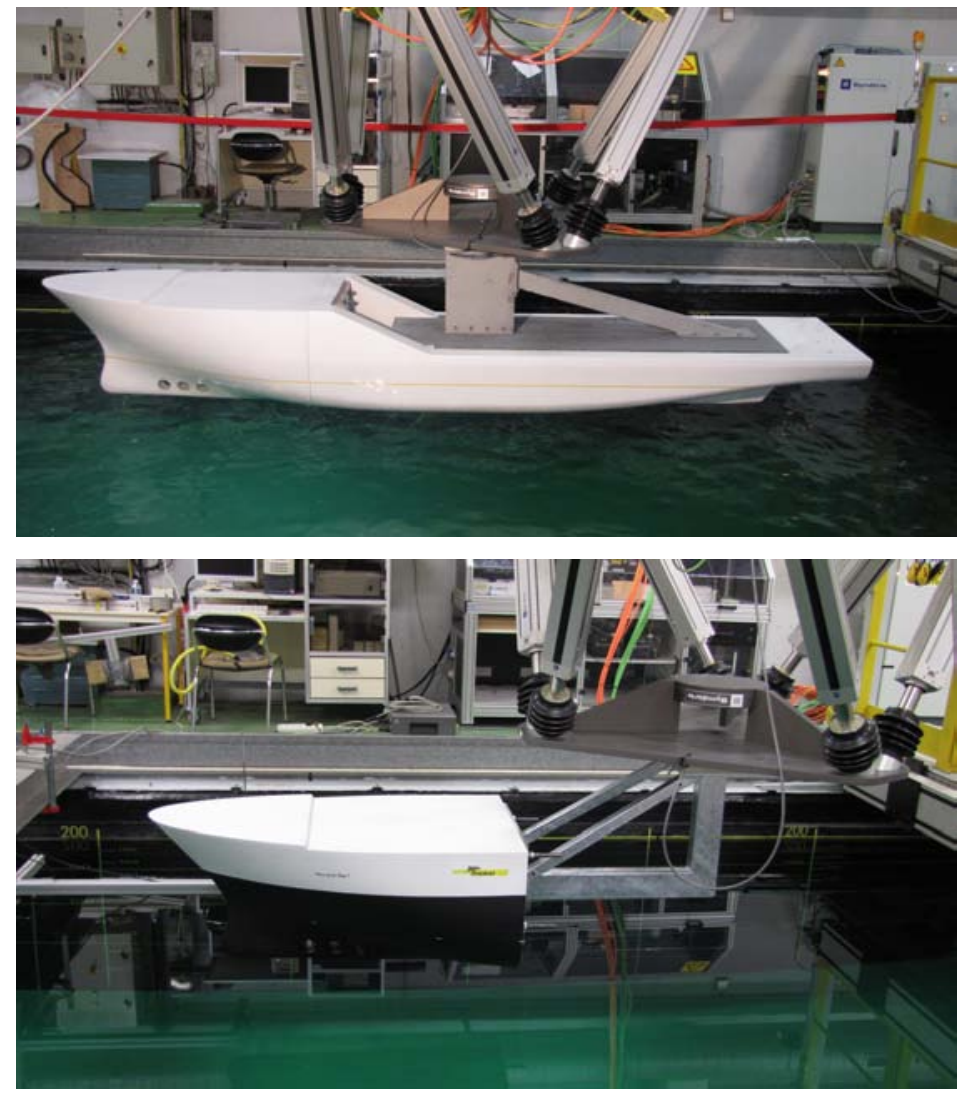

Figure 2: 1/30 model of the Pourquoi pas? fixed under the hexapod. Top: full model. Bottom: bow model only with the same relative position to the hexapod center of reference than for the full model

In the time domain simulation the ship motion components are determined from a set of six coupled differential equations of motion. Integration of the calculated accelerations provides the velocities and a second integration leads to the ship position at each time step.

The model is defined by the geometry, the ship resistance, and the characteristics of the propellers and rudders (see figure 3). Then the conditions of calculation are defined (rotation of the propeller, waves spectrum and direction). The validation of this numerical model is based on the results of the Pourquoi pas? seakeeping tests from MARIN obtained on a 1/16.7 scale model (Technical Report, 2003). The configurations tested with irregular seas are shown in table 1 , where $H_{s}$ is the significant wave height and $T_{p}$ the peak period of the wave spectrum.

Table 1: Configurations tested for the numerical model validation

\begin{tabular}{llllll}
\hline Configuration & 1 & 2 & 3 & 4 & 5 \\
\hline$V[k n o t s]$ & 5 & 10 & 5 & 9 & 5 \\
$H_{s}[m]$ & 3.25 & 3.25 & 5 & 5 & 6.25 \\
$T_{p}[s]$ & 9.7 & 9.7 & 12.4 & 12.4 & 12.4 \\
\hline
\end{tabular}

The results of FREDYN calculations for these configurations are shown in figure 4 . In the 


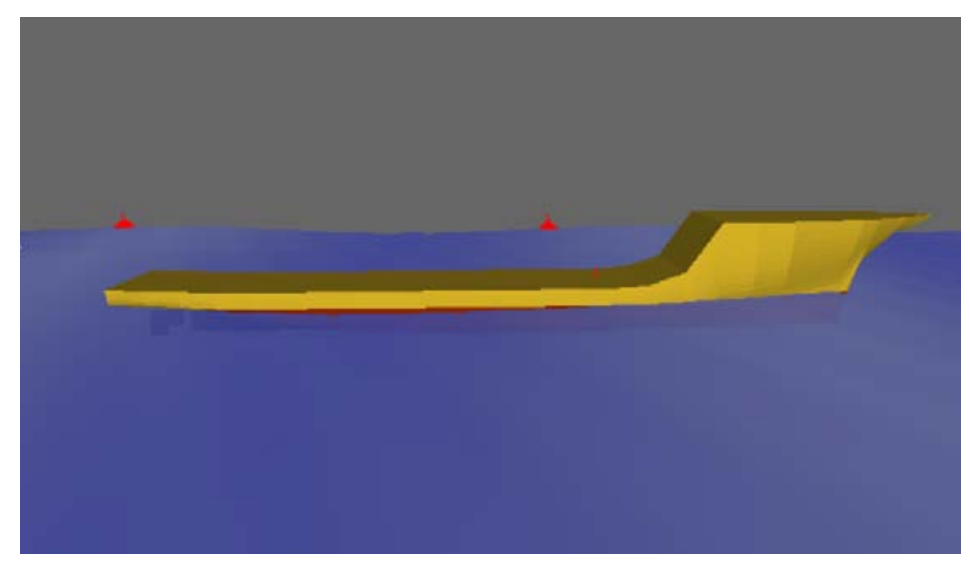

Figure 3: FREDYN numerical model of the Pourquoi pas?

graphic we can see the statistical analysis of pitch obtained for each configuration in terms of standard deviation and significant amplitude (mean of the third highest amplitudes). The pitch is slightly lower for the numerical model for the highest wave amplitude, but the trends are good and the results are very similar for configuration 1 and 2 (respectively $0 \%$ and $8.7 \%$ of error for the standard deviation, and $2.3 \%$ and $1.5 \%$ for the significant amplitude). These configurations are close to the trial conditions, described in section 3 , and validate the numerical model.

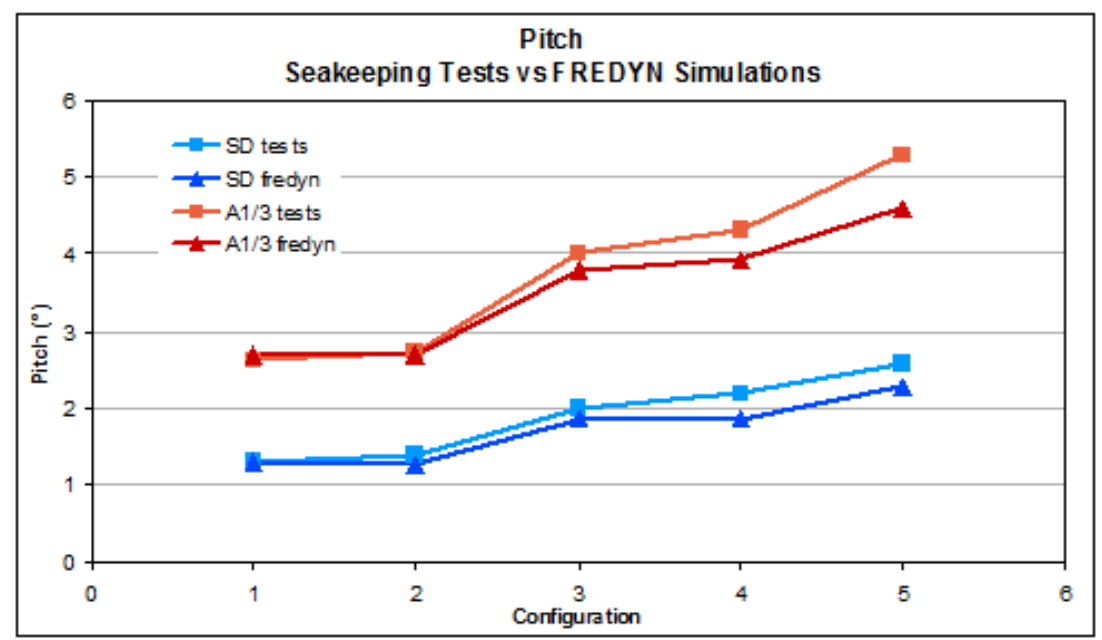

Figure 4: Comparison of Fredyn and seakeeping test results for several sea state conditions

\subsection{Instrumentation}

The first objective of these experiments is to observe and characterize the phenomenon of bubbles generation around the bow. To this aim two synchronized video systems were used: a Tomography system used to obtain very good quality of underwater images, and two high performance digital CMOS cameras for the visualization of the free surface in the bow vicinity. Both systems acquired images at a $15 \mathrm{~Hz}$ frequency.

The tomography system is based on a two-cavities Gemini Nd-Yag laser $(2 \times 120 \mathrm{~mJ} /$ pulse 


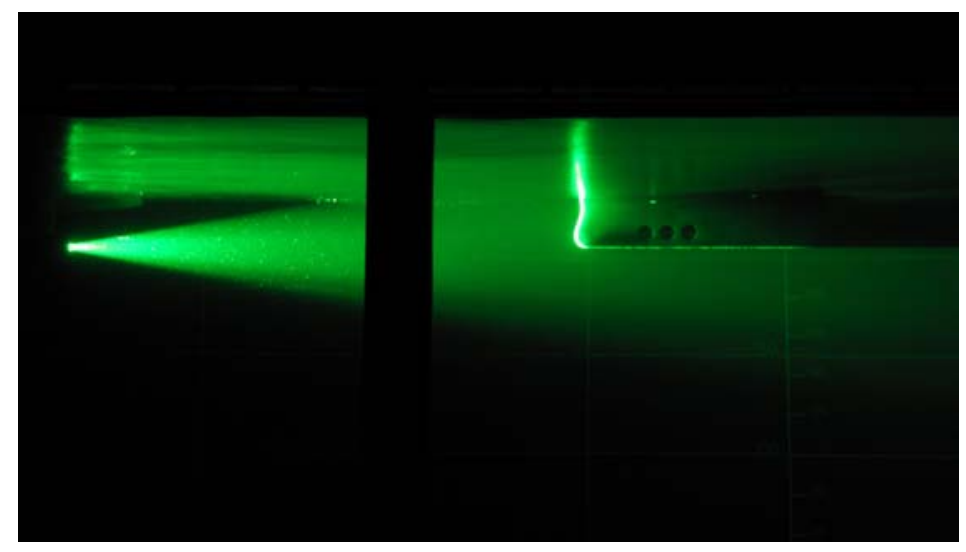

Figure 5: Laser sheet in the ship model axis for the acquisition system in the bow vicinity of the Pourquoi pas?

at $15 \mathrm{~Hz}$ ), using an excitation wavelength of $532 \mathrm{~nm}$. The laser sheet is emitted in the water, by the use of a laser guiding arm in order to generate a light sheet on the vertical plane along the main axis of the ship model (see figure5), far enough of the bow in order to prevent flow perturbations in the measurement area. The camera (Hi-sense CCD camera) records singleframe images in this configuration with a $1600 \times 1200$ pixels $^{2}$ resolution. The distance between the camera -located perpendicularly- and the laser sheet is $2.2 \mathrm{~m}$ so that the image size obtained is $36 \times 27 \mathrm{~cm}^{2}$ with a magnification factor of $0.225 \mathrm{~mm} /$ pixel.

The experimental set-up is summarized in the figure 6 illustrations.

\section{Test configurations}

Before presenting the test parameters and the selected tests configurations, it is necessary to discuss about similarity issues and scale effects. Indeed, these effects are potentially prohibitive for this kind of study, on a 1/30 scale model of an oceanographic vessel in a wave and circulating tank.

\subsection{Similarity issues}

The aim of this work is to have a better understanding of the ship behaviour regarding the generation and propagation of air bubbles. The most important elements involved in these phenomena are waves and ship motions. Therefore, the experimental study requires to observe the Froude similitude $\left(F r=V / \sqrt{g L_{p p}}\right.$ and in this case $\left.F r=0.203\right)$. Hence, a good representation of the free surface influence is ensured. In this case, the first consequence is to work at a different Reynolds number between tests and real scale. Based on the ship speed $V$, and the ship length between perpendiculars $L_{p p}$, the Reynolds number defined as: $R e=V L_{p p} / \nu$ is respectively $R e_{\text {model }}=2.1 \cdot 10^{6}$ in the experiments and $R e_{\text {ship }}=3.4 \cdot 10^{8}$ at full scale. Then the flow would significantly be less turbulent around the model. On the other hand, there is a high turbulent intensity in the tank, due to the presence of the wave generator upstream as detailed in section 2.1. The lower Reynolds number at model scale may affect the boundary layer very 

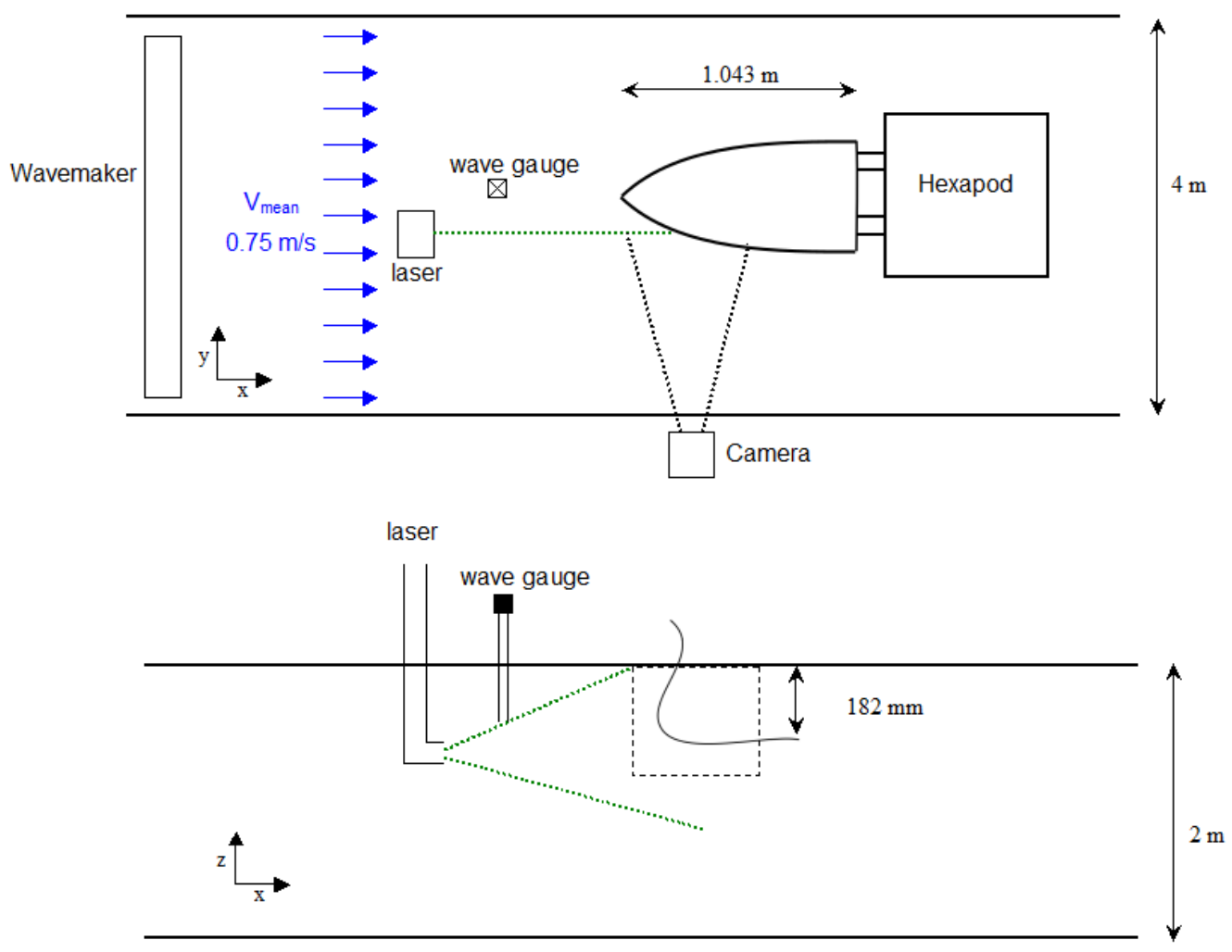

Figure 6: Drawing of the experimental set-up, top and side view showing the light sheet generation system location

close to the hull, but not the propagation of bubble clouds that are generated outside this thin layer (of the order of few $\mathrm{mm}$ ).

Similarity issues are also important for the bubbles behaviour. First of all, the Froude similarity means smaller wave amplitudes, a lower flow velocity and consequently lower energy in the flow around the model. The quantity of air entrapped under the free surface is consequently reduced. Moreover, the size of the bubbles in the flow is determined by a balance between shear stresses, inertia and surface tensions. This issue is associated to the Weber number, defined here with the ship incoming wave amplitude: $W e=\rho A V^{2} / \sigma$, which cannot be conserved during the tests: $W e_{\text {model }}=2.5 \cdot 10^{3}$ in the experiments and $W e_{\text {ship }}=2.3 \cdot 10^{5}$ at full scale. Shear stresses are insufficient to generate microscopic bubbles as can be observed at sea. Several millimetres is the typical size for bubbles in tank, which will have a greater rise velocity. Moreover the salinity of the water directly affects bubble size distribution, limiting coalescence events as explained by Cartmill and Su (1993); Kracht and Finch (2009), increasing the quantity of small bubbles at sea.

All these elements make difficult the observation of bubble sweep-down in classic towing tank as well as direct extrapolation to real scale. However, the original experimental method described below enables a good visualisation and a better apprehension of the phenomenon.

The three significant dimensionless numbers are summarised in table 2 for both model at $1 / 30$ scale and ship at real scale cases. 
Table 2: Main parameters and dimensionless numbers for the tests and at real scale

\begin{tabular}{llllll}
\hline & $L_{p p}(m)$ & $V(m / s)$ & $F r$ & $R e$ & $W e$ \\
\hline Ship & 94 & 4.12 & 0.203 & $3.4 \cdot 10^{8}$ & $2.5 \cdot 10^{3}$ \\
Model & 3.13 & 0.75 & 0.203 & $2.1 \cdot 10^{6}$ & $2.3 \cdot 10^{5}$ \\
\hline
\end{tabular}

\subsection{Test parameters}

Shortly after the first cruises of the Pourquoi pas?, specific tests at sea (Essbulles cruise, 2005) were performed to characterize disturbances on sonar acquisition. These tests showed that such disturbances were due to bubble clouds, confirmed by video measurements from the gondola, and correlated with the pitch in head sea navigation. Bubble sweep-down was observed at sea for the conditions (velocity, wavelength, significant wave height and wave period) given in table 3 .

Table 3: Sailing conditions during the Essbulles cruise, 2005

\begin{tabular}{llll}
\hline$V($ knots $)$ & $\lambda(m)$ & $H_{s}(m)$ & $T_{p}(s)$ \\
\hline 8 & 109 & 2.8 & 8.4 \\
\hline
\end{tabular}

The first step for the experimental study of bubble sweep-down in circulating tank is to reproduce these conditions as close as possible, with current, waves and ship motions. In this case, ship motions are imposed by the use of an hexapod, synchronized with the wave generator. To this end, it is necessary to work with regular waves. In these conditions the uncertainty of the waves and resulting motions is suppressed, which prevents extreme events. The characteristics of the regular waves will be calculated to conserve the energy density of the conditions encountered at sea.

The energy density of irregular waves is given by the following expression:

$$
E=\rho g m_{0}\left[J / m^{2}\right], \text { with } H_{s}=4 \sqrt{m_{0}}
$$

Where $m_{0}$ is the zero order moment of the wave energy spectra.

For sinusoidal waves:

$$
E=\frac{1}{2} \rho g A^{2}, \text { where } \mathrm{A} \text { is the amplitude }(A=H / 2) .
$$

To observe energy density conservation, one must write:

$$
\begin{gathered}
m_{0}=\frac{1}{2} A^{2} \\
\text { so } A=\frac{\sqrt{2} H_{s}}{4} \text { and } H=\frac{\sqrt{2} H_{s}}{2}
\end{gathered}
$$

For $H_{s}=2,8 \mathrm{~m}$ the sinusoidal waves with the same energy density will have a wave height 
$H=2,0 \mathrm{~m}$. The period $T$ will be taken equal to $T_{p}$.

The input characteristics of the regular waves for the wave generator are the amplitude and the frequency. The current and the wave amplitude for the trials are directly obtained by the Froude scaling. At the model scale the ship speed is $U=V / \sqrt{30}=0.75 \mathrm{~m} / \mathrm{s}$. The wave amplitude is $A_{m}=A_{r} / 30=33 \mathrm{~mm}$. For the calculation of the frequency it must be taken into account that the model is fixed and the encounter frequency is higher than that of the wave. The encounter frequency is given by:

$$
f_{e}=f-\frac{V}{\lambda} \cos (\theta)
$$

with $\theta=0^{\circ}$ for following sea and $180^{\circ}$ for head sea. So in our configuration:

$$
f_{e}=f+\frac{V}{\lambda}
$$

We finally obtain $f_{e}=0,85 \mathrm{~Hz}$, and the final trial conditions are summarized in table 4 . The generation of waves in the tank is illustrated on figure 7 .

Table 4: Trial main parameters

\begin{tabular}{lll}
\hline$U(\mathrm{~m} / \mathrm{s})$ & $A(\mathrm{~mm})$ & $f_{e}(\mathrm{~Hz})$ \\
\hline 0.75 & 33 & 0.85 \\
\hline
\end{tabular}

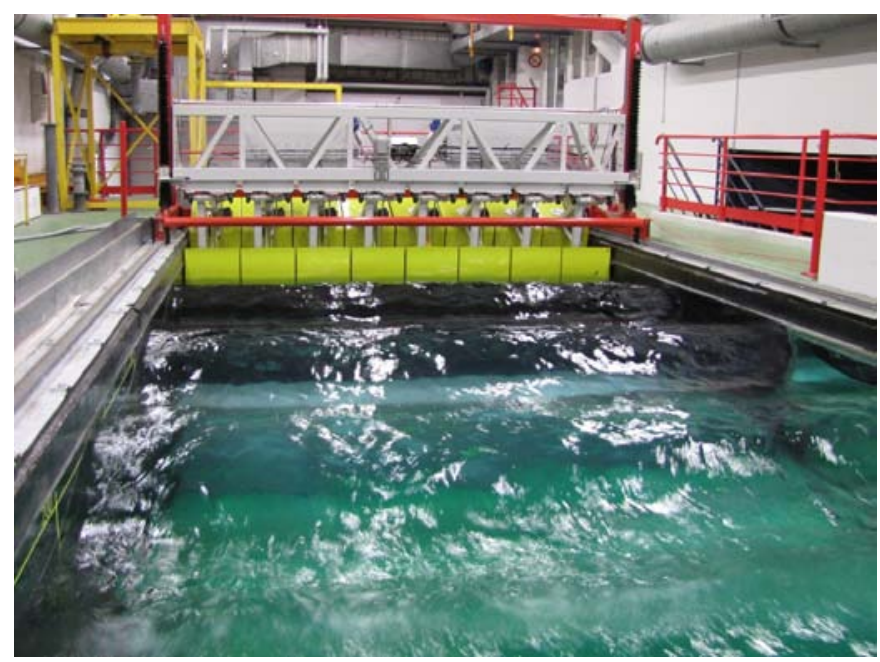

Figure 7: Illustration of regular wave generation in the tank with a current of $0.75 \mathrm{~m} / \mathrm{s}$

After the numerical model validation and the determination of trial conditions, ship motions are calculated with the use of the Fredyn model. The calculation inputs correspond to regular waves of $2.0 \mathrm{~m}$ height and $8.4 \mathrm{~s}$ period and a ship velocity of $8 \mathrm{knots}$. The full scale results are shown on figure 8. Surge movements, not represented on the graphic, are insignificant $(<0.1 \mathrm{~m}$ at full scale) and will not be taken into account during this study. In this head sea configuration, the only significant movements are the heave and the pitch. It must be emphasized here that 
wave and heave are in-phase.

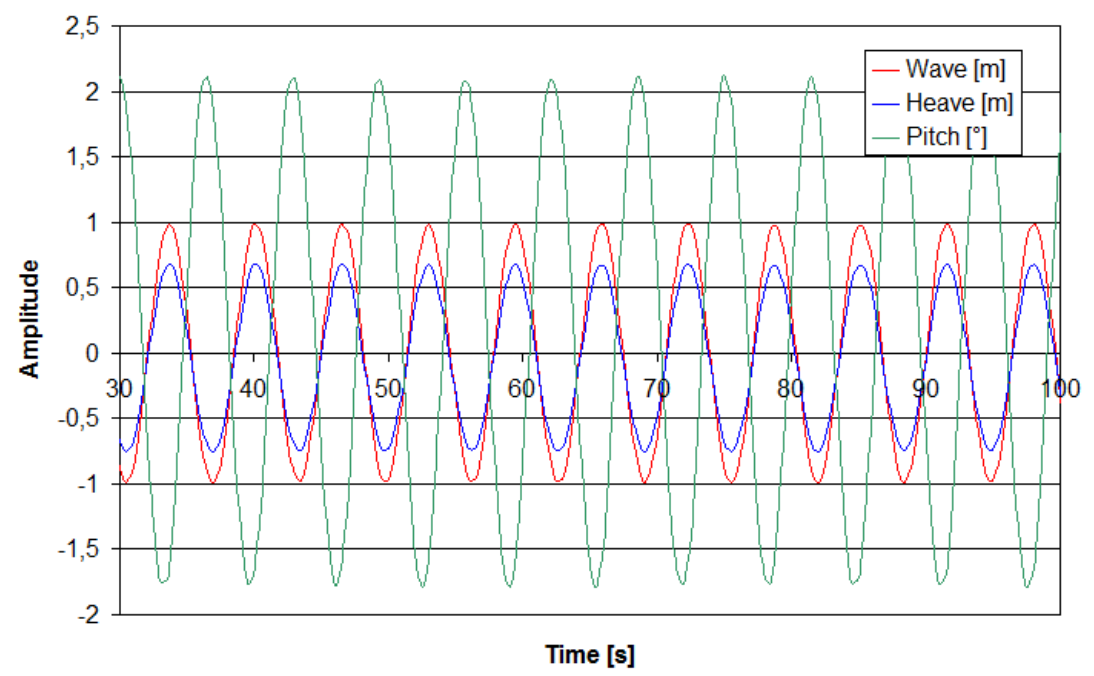

Figure 8: FREDYN results for the trial conditions at real scale

These results must be converted to model scale by Froude scaling $(\times 1 / \sqrt{30}$ for the time, $\times 1 / 30$ for the heave and $\times 1$ for the pitch). It is finally obtained $2^{\circ}$ pitch and $20 \mathrm{~mm}$ heave model motion amplitudes.

\subsection{Wave and motion synchronism}

A key point of the methodology is the synchronisation of the waves and motions. For that purpose a start-up procedure has been developed and is summarized in figure 9 . The user starts the wave generator sequence, which sends a trigger signal that starts the hexapod sequence. In this sequence a pause time must be inserted for the wave to reach the model. The phase shift between wave and motion is adjusted by this pause time. The hexapod sequence also sends a trigger signal that starts simultaneously all measurement systems (laser, cameras, motions acquisition system, waves gauges)

The synchronism is verified a posteriori with the motions and wave recording as can be seen on figure 10. The free surface elevation at the model center of gravity is obtained from measurement $1 \mathrm{~m}$ upstream the bow. Previous tests with 2 wave gauges were undertaken to calculate the wave celerity.

Motions generated by the hexapod as well as wave frequency are extremely precise while wave amplitude fluctuations are important due to the interaction between the current and the wave generator. The standard deviation of the wave amplitude is $13 \mathrm{~mm}$ and less than $0.1 \mathrm{~mm}$ for the measured heave amplitude. The mean phase shift is calculated on the whole sequence, and only the sequences with less than $10^{\circ}$ of mean phase shift for which there is no influence on bubble generation occurrence are conserved for the analysis. Because the wave amplitudes fluctuate but motions don't, the imposed motions calculated with Fredyn are not reproducing the proper motion for a free model on every period. It will result in more violent forces in some periods, inducing more bubble entrainment, or the opposite at other periods. For such reasons many analysis of bubbles clouds generation were performed on single periods separately, selected 


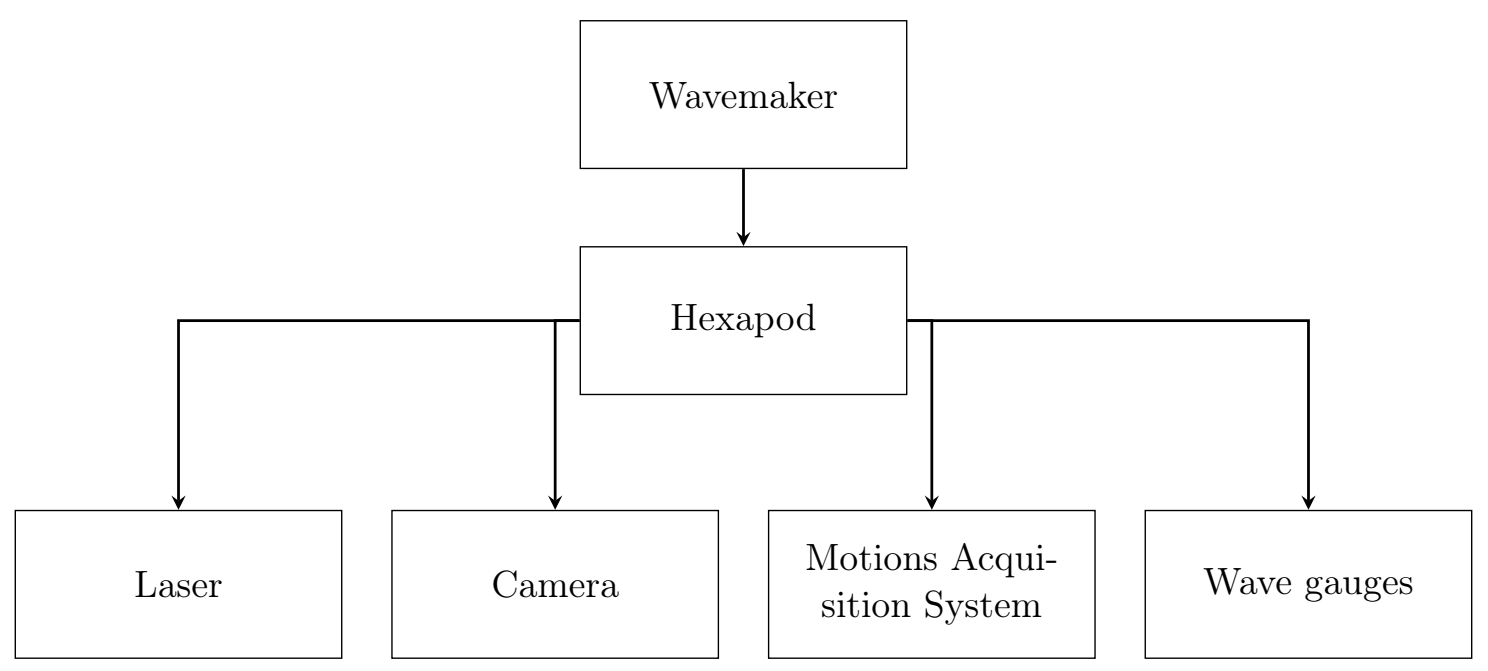

Figure 9: Synchronism diagram of the experimental set-up between the wavemaker, the hexapod and the dedicated instrumentation

by the wave amplitude. A wave by wave analysis is preferable than a statistical one.

\subsection{Test configurations}

The final objective of this work is to understand bubbles generation and propagation around the hull, to be able to offer solutions to minimize the phenomenon. Several configurations have been tested to apprehend the influence of each parameter.

- In the first configuration (case 1), the model is fixed and only submitted to the circulating tank current $(U=0.75 \mathrm{~m} / \mathrm{s})$.

- In the second configuration (case 2), the model is fixed and submitted to current and regular waves (33 $\mathrm{mm}$ amplitude and $0.85 \mathrm{~Hz}$ frequency).

- In the third configuration (case 3), motions are forced by the hexapod ( $2^{\circ}$ pitch and $20 \mathrm{~mm}$ heave at $0.85 \mathrm{~Hz}$ ), and the model is submitted to current.

- In the fourth configuration (case 4), the model is submitted to current, as well as synchronized waves and motions.

\section{Bubble sweep-down phenomenon}

In this section, we first describe the phenomena observed through the experimental method developed above, from observations of the free surface behaviour at the bow, and of the bubble clouds in the water column. Secondly, bubble clouds occurrence is discussed for the four configurations.

\subsection{Visualization of bubble generation}

Through the experimental configurations described in section 3, two different air entrainment mechanisms have been detected. In the following these two kinds of bubble cloud will be refered 


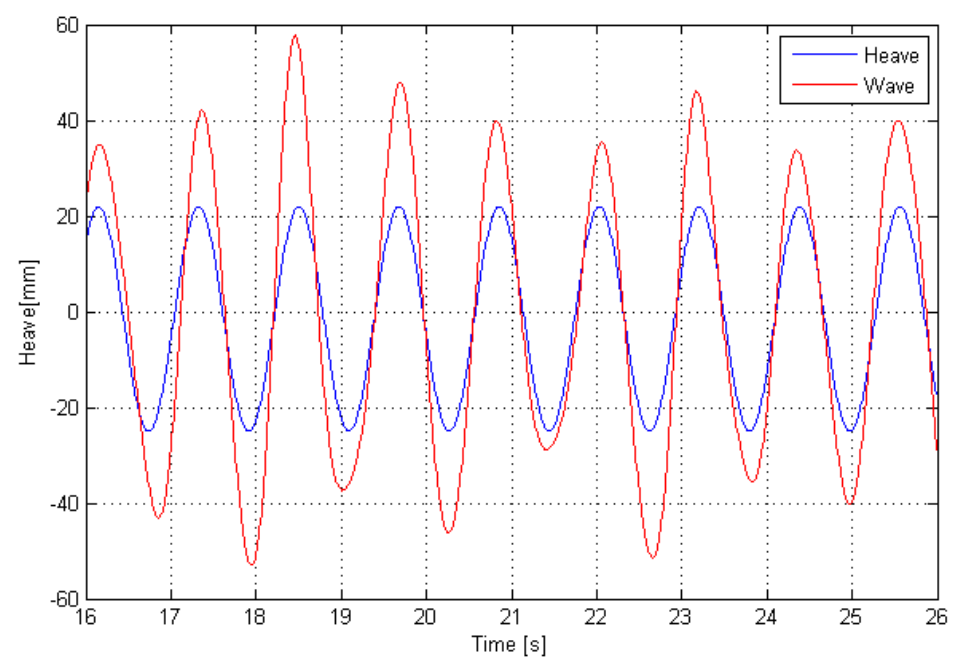

Figure 10: Exemple of waves and heave synchronisation from the experimental measurements with a current of $0.75 \mathrm{~m} / \mathrm{s}$

respectively as vortex shedding cloud and breaking wave cloud. These phenomena are completely independent and easy to distinguish, even if for very few cases both air entrainment mechanisms can occur at the same time. For these particular events, we are also able to distinguish each of them.

The first kind of air entrainment is encountered in all configurations, even without waves and motions. In this case, the incoming turbulent flow generates a distortion of the free surface in contact with the bow. A small air cavity is developed until the air entrapment by vortex shedding. The initiation of this phenomenon may be held for several tenths of seconds. This phenomenon appears randomly throughout the test sequence, but with a stable frequency of occurrence. A schematic description of the vortex entrainment mechanism is presented on figure 11.

The formation of the cavity occurs when the flow shows a high velocity gradient at the bow contact, with plunging and ascending speeds relatively close. When the velocity gradient becomes too large, the cavity widens and becomes unstable. Then the appearance of a vortex may be observed (whose main axis is transverse to the measurement plane), causing air entrainment and the generation of the corresponding bubbles cloud.

The second kind of air entrainment is due to breaking waves, coming from the interaction of several factors depending on the configuration: the incoming wave and a reflected one on the bow and/or the impact between the bow and the free surface. The breaking phenomena observed in configurations 2, 3 and 4 are similar. Only the magnitude and the frequency is changing with the configuration. The air entrainment by a breaking wave is more energetic and almost instantaneous. It can be seen on figure 12 that bubbles do not pass under the hull. As detailed in section 3.1, the bubbles generated around the model are on the range of several millimetres and rise to the surface rapidly. These mechanisms observed in laboratory are similar to those encountered at sea, even if the quantity of air entrained and the size of bubbles are distinct.

The synchronism between the different cameras allows to correlate the free surface phe- 

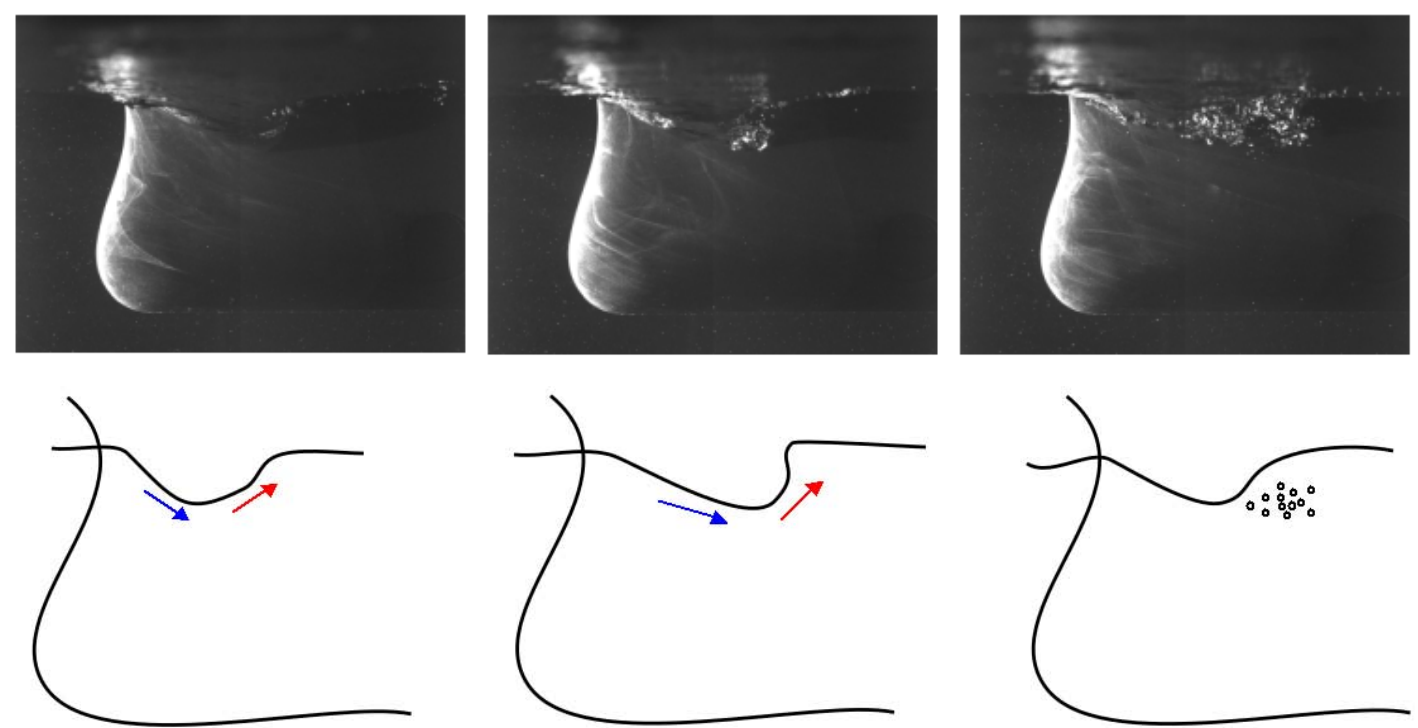

Figure 11: Description of the vortex shedding clouds generation from the free surface distortion to the flow aeration, encountered in the fourth cases

nomenon and the generation of bubbles as can be seen on figures 13 and 14 for the breaking wave cloud case. On these figures are represented six instants of the air entrainment process acquired underwater (left images) and above the free surface by one of the two CMOS cameras (right images). The time interval between each moment is two images $(0.13 s)$. The corresponding pitch, heave and free surface elevation at the center of gravity are represented on figure 15 .

The three instants (t1, t2 an $\mathrm{t} 3$ ) of figure 13 correspond to a peak of pitch. The front of the model impacts the free surface and the incoming wave, generating a breaking bow wave. The bubbles that can be seen on these pictures are due to a previous vortex shedding cloud. The bow wave is entirely formed at $\mathrm{t} 3$, and the impact occurs before $\mathrm{t} 4$.

On figure 14 ( $\mathrm{t} 4, \mathrm{t} 5$ and $\mathrm{t} 6$ ) the bow is pulled up, and air is entrapped by the breaking wave. The bubble cloud formed is entrained down by the plunging jet velocity at the instant when the bow is highest.

The detail of images analysis leading to bubble clouds surface and density, bubble depth penetration, clouds velocities, for both breaking wave and vortex shedding cases will be given in the second part of the article.

\subsection{Bubble clouds frequency}

The frequency of each air entrainment mechanism for each configuration can be determined from video analysis of the underwater sequences directly by operators visualization (several operators have realized these analysis and obtain the same bubble clouds occurrence). The results obtained for the four cases are shown on figure 16. In the first case with current only, the bubble cloud frequency is low $(<0.2 \mathrm{~Hz})$ and only due to vortex shedding along the hull. This phenomenon is caused by the interaction between the bow shape and the highly turbulent incoming flow. In 

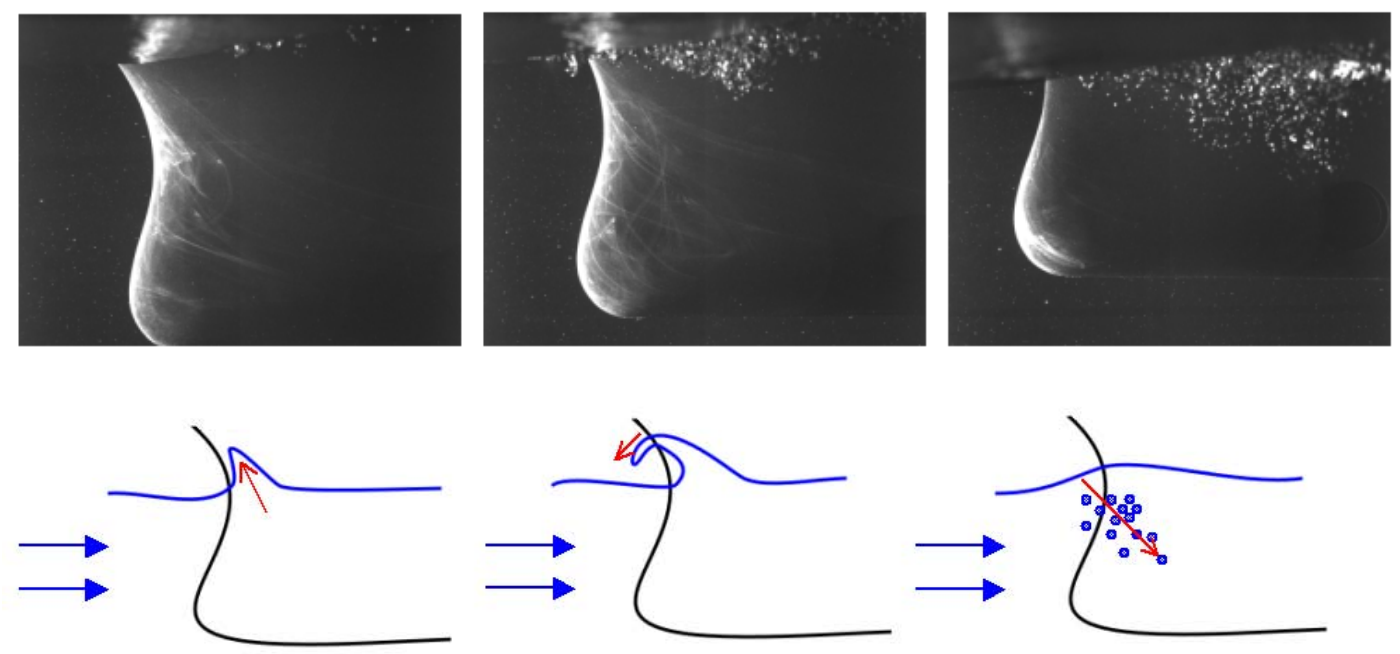

Figure 12: Description of the breaking wave clouds generation from the generation of a reflective wave on the bow to the flow aeration, encountered only for case 2 to 4

the second case with waves and current, breaking bow waves were observed. The proportion of bubble clouds generation increases to reach a cloud generation seven out of ten waves $(0.59 \mathrm{~Hz}$ for clouds frequency compared to $0.85 \mathrm{~Hz}$ for the wave frequency). Here the two kinds of bubble clouds are encountered with approximately the same level of occurrence of vortex shedding cloud type as for the case 1. The breaking wave cloud type appears twice more frequently than the other one. For the case of hull motion under current (case 3), the bubble cloud occurrence is approximately twice than for case 1 . Here also the two kinds of bubble clouds are encountered with similar level of occurrence for each of them. The hull motions generate less bubble clouds than waves (a cloud frequency of $0.40 \mathrm{~Hz}$ compared to $0.59 \mathrm{~Hz}$ ). For the hull motion under waves and current (case 4), the combination of solicitations leads to an accumulation of effects until the generation of bubble cloud for nearly eight out of ten incoming wave $(0.66 \mathrm{~Hz})$. In this case, breaking wave cloud type is mainly encountered.

These frequency calculations were undertaken on 5 minutes sequences ( 250 wave periods). Air entrainment frequency were also calculated every 30 seconds in order to confirm the convergence of the results. Figure 17 shows this process for the four configurations. In the second part of the article, the influence of the tests parameters will be analysed (wave or motion amplitude and frequency for instance). For all these trials the recording sequences will be limited to 3 minutes (150 wave periods) and considered to be converged, thanks to the stability of the mean values obtained on longer periods as shown on figure 17.

\section{Conclusion}

The difficulty of observing bubble sweep-down on ship models during towing tank test led us to develop an original method to study this phenomenon. We demonstrate in this paper that a wave and current circulating tank is particularly well suited to achieve this goal. Even if scale effects discussed in section 3.1 are significant, this methodology allows the quantification 
of the phenomena leading to bubble generation around the bow of a ship under waves and current. The observation window gives an easy and good view of the phenomenon. The use of the motion generator enables the study of wave and ship movement independently, while the specific turbulent current is a source of air entrainment. The methodology developed in this paper also allows to work on the bow model only, which was a significant progress for the study in such facility. In this configuration it was possible to observe simultaneously the free surface behaviour and the generation of bubbles. Two distinct mechanisms of air entrainment were encountered and described. The vortex shedding bubble clouds appear randomly in all the configurations tested, even without waves or motions. This phenomenon is due to the interaction between the turbulent flow and the specific bow shape of the Pourquoi pas?. On the other hand the breaking wave clouds appear in presence of relative motions between the free surface and the bow ship and more significantly under wave sollicitations.

In the second part of the article, image analysis will be used to analyse in detail these two kinds of bubble clouds formation. The impact of several parameters will be studied to understand the influence of each of them on the bubble generation. Therefore the influence of wave amplitude and frequency, motions amplitude and frequency, current speed, turbulence, phase shift between waves and motions will be studied.

Such results could be used for the validation of future numerical models searching to consider these phenomena. Moreover the instrumentation used in this method can be also involved in the acquisition of velocity maps of the flow around the bow, that are representative of real scale through the Froude similarity. By this way different bow shapes performances regarding the bubble sweep-down phenomena could be compared.

\section{Acknowledgements}

The authors gratefully acknowledge the DGA (the French Government Agency for Defense) and Ifremer for the financial support of co-financed $\mathrm{PhD}$ these.

We also would like to thank Thomas Bacchetti and Jean-Valery Facq for their assistance in the design and set-up of these experiments.

\section{References}

Bouhoubeiny E., Druault P.,Germain G., 2014, Phase-average mean properties of turbulent flow developing around a fluttering sheet of net, Ocean Engineering, Vol.82, 160-168.

Carrica P.M., Castro A.M.,Li J., Politano M.,Hyman M., 2012, Towards an air entrainment model, Proc. of the $29^{\text {th }}$ Symposium on Naval Hydrodynamics, Gotheburg, Sweden.

Castro A.M., Li J., Hyman M., Carrica P.M., 2014, Turbulent and cavity free surface bubble entrainment with application to ship hydrodynamics, Proc. of the $30^{\text {th }}$ Symposium on Naval Hydrodynamics, Hobart, Tasmania, Australia.

Cartmill J.W. and Su M.Y., 1993, Bubble size distribution under saltwater and freshwater breaking waves, Dynamics of Atmosphere and Oceans, Vol.20, 25-31. 
Chanson H. and Cummings P.D., 1994, Effects of Plunging Breakers on the Gas Contents in the Oceans, Marine Technology Society Journal, Vol.28, 22-32.

Cooper E.B., 2012, The future RRS Discovery, Ocean Challenge, Vol.19, 9-10.

Dalen J. and Løvik A., 1981, The influence of wind-induced bubbles on echo integration surveys, J. Acoust. Soc. Am., Vol.69, 1653-1659.

De Kat J.O. and Paulling J.R., 2001, Prediction of Extreme Motions and Capsizing of Ships and Offshore Vehicles, Proc. of the $20^{\text {th }}$ OMAE Conference, Rio de Janeiro.

Delacroix S., 2015, Caractérisation de la génération et de la propagation de bulles autour de la carène de navires scientifiques, PhD Thesis, Université de Bretagne Occidentale.

Delacroix S., Germain G., Berger L., Billard JY., 2016, Bubble sweep-down occurrence characterization on Research Vessels, Ocean Engineering, Vol. 111, 34-42.

Delhommeau G., Guilbaud M., David L., Yang C., Noblesse F., 2009, Boundary between unsteady and overturning ship bow wave regimes, Journal of Fluid Mechanics, Vol.620, 167-175.

Deane G.B. and Stokes M.D., 1999, Air entrainment processes and bubble size distributions in the surf zone, J. Phys. Oceanogr., Vol.29, 1393-1403.

Deane G.B. and Stokes M.D., 2002, Scale dependence of bubble creation mechanisms in breaking waves, Nature, Vol.418, 839-844.

Gaurier B., Davies P., Deuff A. and Germain G., 2013, Flume tank characterization of marine current turbine blade behaviour under current and wave loading, Renewable Energy, Vol.59, $1-12$.

Kracht W. and Finch J.A., 2009, Bubble break-up and the role of frother and salt, Int. J. Miner. Process., Vol.92, 153-161.

Ma J., Oberai A.A., Hyman M.C., Drew D.A., Lahey R.T. Jr, 2011, Two-fluid modeling of bubbly flows around surface ships using a phenomenological subgrid air entrainment model, Computers \& Fluids, Vol.52, 50-57.

MARIN Technical report, Seakeeping tests for the research vessel "Pourquoi pas?" Final report, Report No 18268-2-SMB.

Moraga F.J., Carrica P.M., Drew D.A., Lahey R.T. Jr, 2008, Air entrainment model for breaking bow waves and naval surface ships, Computers \& Fluids, Vol.37, 281-298.

New A.L., 1992, Factors affecting the quality of shipboard acoustic Doppler current profiler data, Deep-Sea Research, Vol.39, 1985-1996.

Noblesse F., Delhommeau G., Guilbaud M., Hendrix D., Yang C., 2008, Simple analytical relations for ship bow waves, Journal of Fluid Mechanics, Vol.600, 105-132. 
Noblesse F., Delhommeau G., Guilbaud M., Liu H., Wan D., Yang C., 2013, Ship bow waves, Journal of Hydrodynamics, Vol.25, 491-501.

Rolland D. and Clark P., 2010, Reducing bubble sweepdown effects on research vessels, OCEANS'10 IEEE Sydney.

Shabangu F.W., Ona E. and Yemane D., 2014, Measurements of acoustic attenuation at $38 \mathrm{kHz}$ by wind-induced air bubbles with suggested correction factors for hull-mounted transducers, Fisheries Research, Vol.151, 47-56.

Shakeri M., Tavakolinejad M. and Duncan J. H., 2009, An experimental investigation of divergent bow waves simulated by a two-dimensional plus temporal wave marker technique, Journal of Fluid Mechanics, Vol.634, 217-243.

Tavakolinejad M., 2010, Air bubble entrainment by breaking bow waves simulated by a $2 \mathrm{D}+\mathrm{T}$ technique, PhD Thesis, University of Maryland.

Trevorrow M.V., Measurements of near-surface bubble plumes in the open ocean with implications for high-frequency sonar performance, J. Acoust. Soc. Am., Vol.114, 2003.

Von Bröckel K., 2014, Echo Sounders versus Air Bubbles in Research Vessels, Hydro International, January/February, 14-17.

Waniewski T.A., Brennen C.E. and Raichlen F., Measurements of Air Entrainment by Bow Waves, Journal of Fluids Engineering, Vol.123, 57-63. 

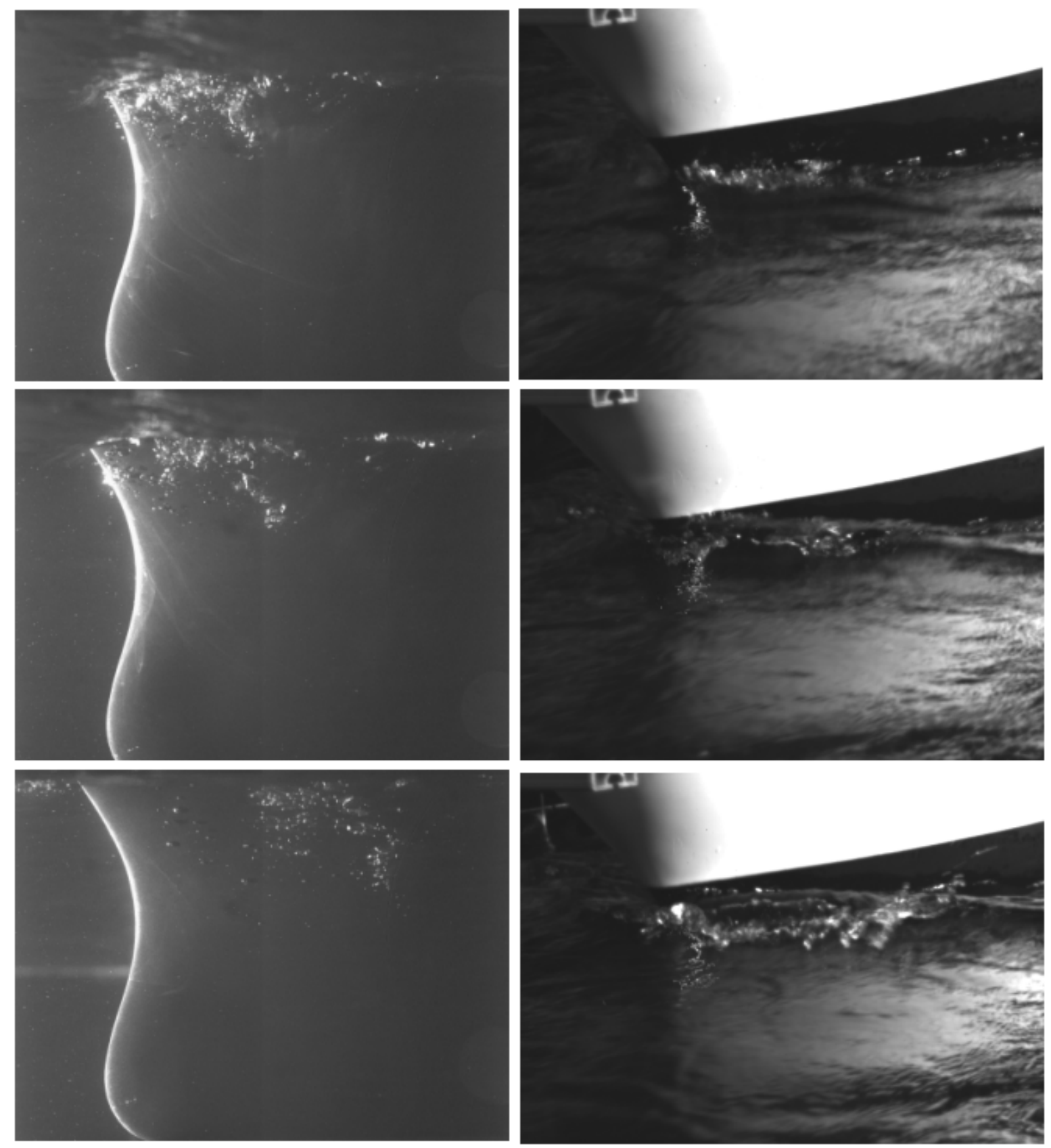

Figure 13: Simultaneous visualizations of the generation of bubbles (left) and the breaking wave (right) during the breaking wave event (t1, t2 and $\mathrm{t} 3$ ). 

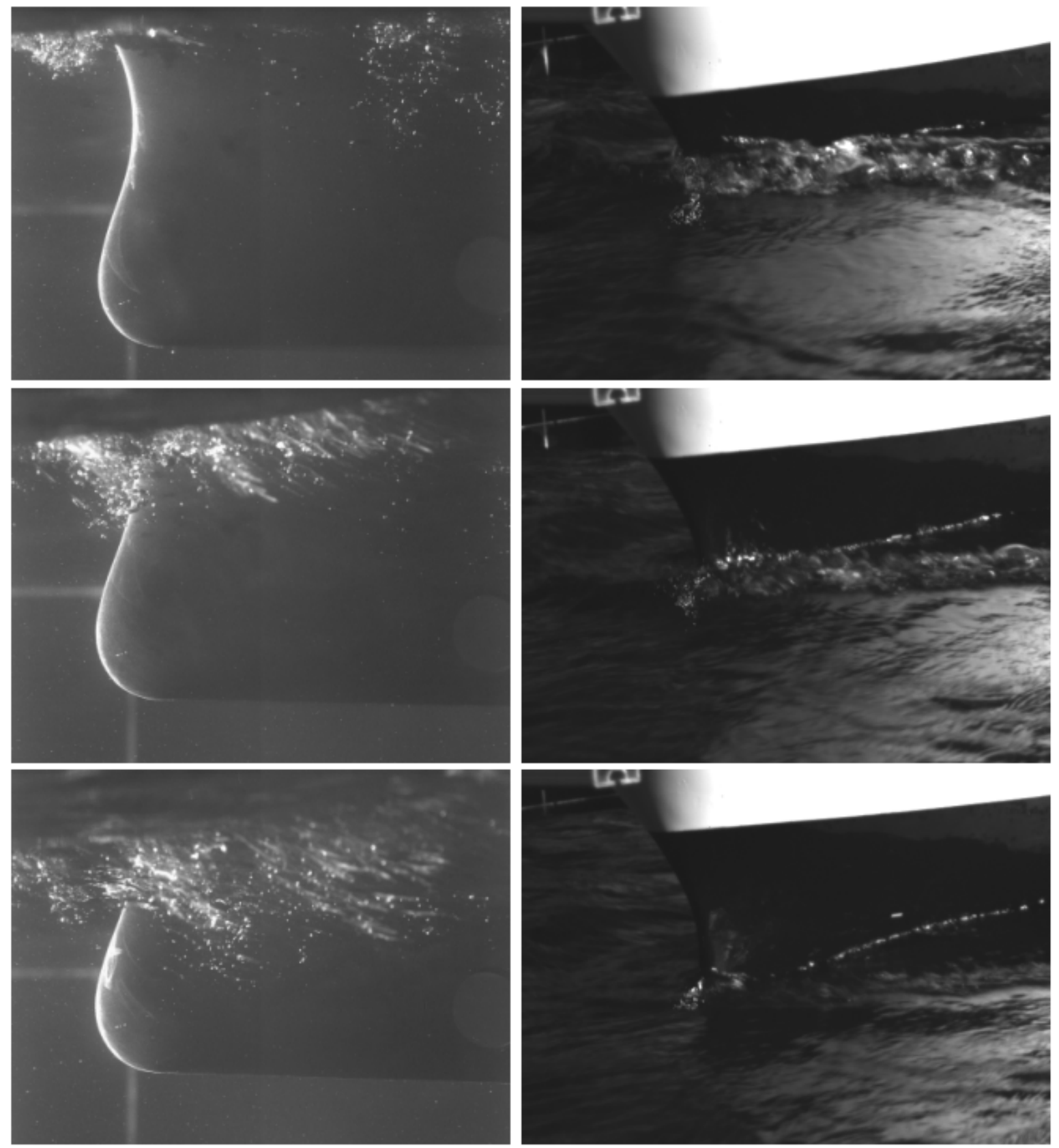

Figure 14: Simultaneous visualizations of the generation of bubbles (left) and the breaking wave (right) during the breaking wave event ( $\mathrm{t} 4, \mathrm{t} 5$ and $\mathrm{t} 6$ ). 


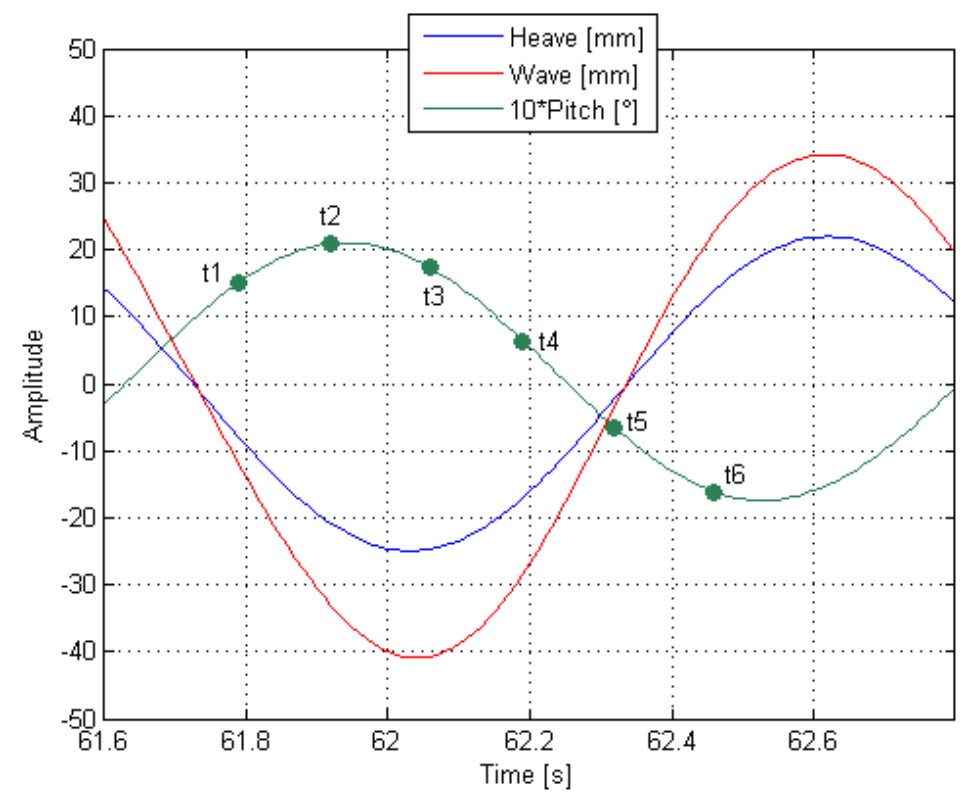

Figure 15: Pitch, heave and free surface elevation (at the center of gravity) during instantaneous visualizations $(\mathrm{t} 1 \rightarrow \mathrm{t} 6)$ of breaking wave and air entrainment events.

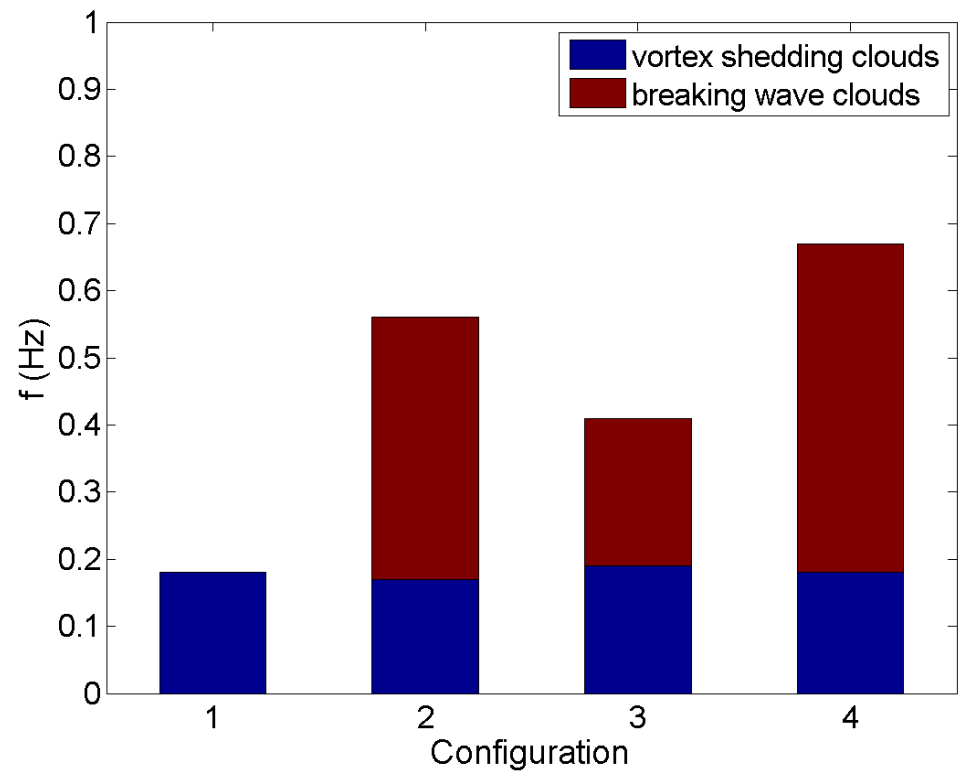

Figure 16: Air entrainment frequency, breaking wave clouds and vortex shedding clouds, for the 4 test configurations 


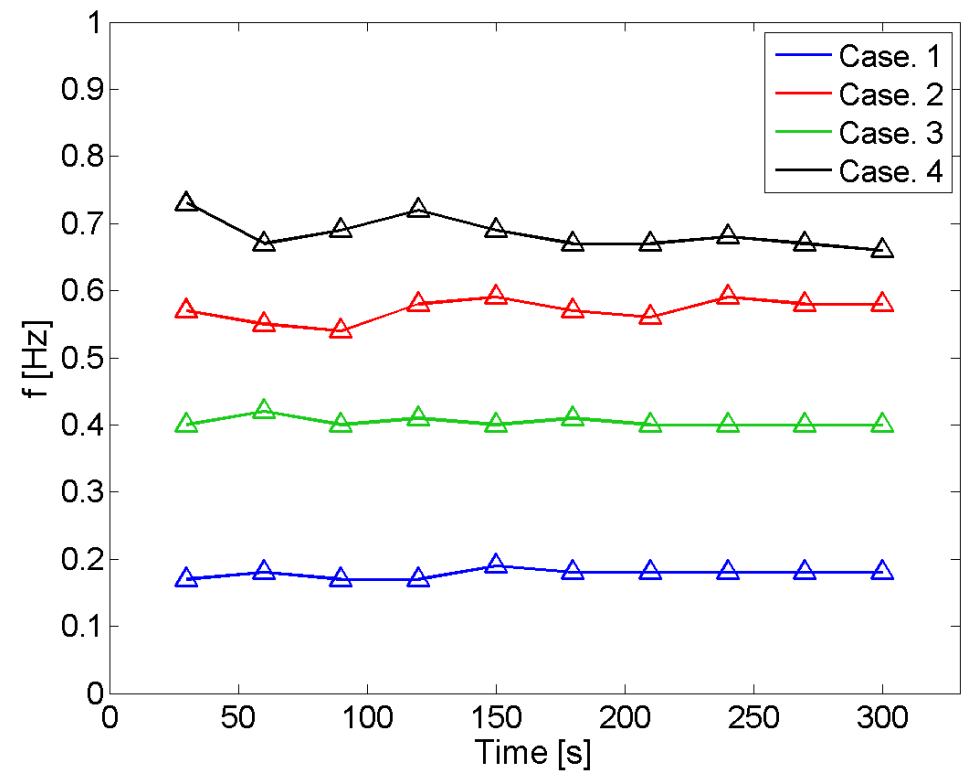

Figure 17: Study of bubble clouds frequency convergence for the 4 test configurations 